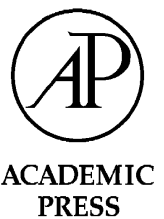

Available online at www.sciencedirect.com

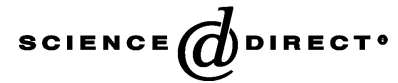

Journal of Sound and Vibration 263 (2003) 113-129

\title{
Analytical evaluation of the acoustic insulation provided by double infinite walls
}

\author{
J.M.P. António*, A. Tadeu, L. Godinho \\ Department of Civil Engineering, University of Coimbra, Polo II-Pinhal de Marrocos, 3030-290 Coimbra, Portugal
}

Received 31 October 2001; accepted 10 May 2002

\begin{abstract}
The acoustic insulation provided by infinite double panel walls, when subjected to spatially sinusoidal line pressure loads, is computed analytically. The methodology used extends earlier work by the authors on the definition of the acoustic insulation conferred by a single panel wall. It does not entail any simplification other than the assumption that the panels are of infinite extent. The full interaction between the fluid (air) and the solid layers is thus taken into account and the calculation does not involve limiting the thickness of any layer, as the Kirchhoff or Mindlin theories require. The problem is first formulated in the frequency domain. Time domain solutions are then obtained by means of inverse Fourier transforms using complex frequencies.

The model is first used to compute the sound reduction provided by a double homogeneous brick wall, with identical panels, when illuminated by plane sound waves. The results are then compared with those provided by the simplified method proposed by London, which was later extended by Beranek (LondonBeranek method). The limitations of the simplified London-Beranek model, namely, its applicability only to double walls with identical mass, subjected to plane waves, and its failure to account for the coincidence effect, are overcome by the method proposed.

Time signatures are produced to illustrate the different sound transmission mechanisms. Several types of body and guided waves are originated, giving rise to a complex dynamic system with multiple reflections within the solid and fluid layers and the global resonance of the system. The effect of the cavity absorption is considered by attributing a complex density to the air filling the space between the two wall panels. Absorption attenuates the dips of insulation controlled by the cavity resonances. Several simulations are then performed for different combinations of wall and air layer thickness to assess the influence of this variable on the final acoustic insulation. The influence of the air cavity on sound reduction was found to be dependent on the frequency. At low frequencies a better performance was achieved for thicker air layers, while at higher frequencies a thinner air layer is preferable. The use of wall panels with different mass resulted in the wall performing better, particularly for high frequencies.
\end{abstract}

(C) 2002 Elsevier Science Ltd. All rights reserved.

\footnotetext{
*Corresponding author. Tel.: +351-239-797196; fax: +351-239-797190.

E-mail address: julieta@dec.uc.pt (J.M.P. António).
} 


\section{Introduction}

Different dynamic mechanisms are involved in the transmission of sound energy through a solid double wall. Several types of body and guided waves are originated, giving rise to a complex dynamic system. In this system some of the relevant variables are the mass, the density, the elasticity modulus and the Poisson coefficient of the material of each panel, the air space separating the two walls, the effect of the cavity absorption, the frequency of excitation and the type of source.

The acoustic insulation is highly dependent on both the mass of each wall and the excitation frequency of the pressure source. As the mass of each wall increases, so does insulation, due to increasing inertial forces. When the frequency of sound incident on a wall that maintains the same mass is increased, the vibration power of the element decreases and greater dissipation of sound energy occurs, leading to a rise in acoustic insulation.

Different simplified predictive insulation models have been built that only take a limited number of variables into account. In the case of an infinite single panel, the sound reduction index is given by the theoretical law of frequency, or mass, which assumes the element to behave like a group of infinite juxtaposed masses, with independent displacement, and null damping forces [1]. Novikov [2] tried to approach the sound insulation of finite plates at low excitation frequencies by adding a correction coefficient to the mass law. However, the theoretical frequency and mass law does not predict the dips of insulation that occur when the response of the dynamic system is larger. This happens particularly when the system vibrates in the vicinity of eigenmodes, such as those caused by the coincidence effect.

In the presence of a double wall, the problem becomes more complex because of the dynamic vibration of each panel, coupled with the vibration of pressure waves within the air gap between the two panels and with the exterior air medium. London [3] has solved the case of sound transmission through double walls, when both walls are identical. In this model the double wall is excited by plane waves at frequencies below their critical frequency, and its mass is controlled so that the panel resonances could be disregarded. This simplified equation takes into account the dips of insulation caused by the multiple reflections within the air chamber, which corresponds to the acoustic resonance of the cavity. Further mathematical manipulation of this equation allows the global basic resonance (mass-air-mass resonance) to be considered [4]. The effect of the cavity absorption provided by filling the air cavity with sound-absorbing material has been considered in different models. Gösele [5] proposed a simplified method to predict the sound transmission loss through a double wall, without structure-borne connections and where the gap is filled with porous sound-absorbing material, when the measured sound transmissions of the two constituent single partitions are available. Fahy [6] simulated this effect by using a complex air density, which is dependent on the flow resistivity and the porosity of the material, and on a structure factor.

Different numerical techniques such as the statistical energy analysis method (SEA), the finite element method (FEM) and the boundary element method (BEM) have been formulated and implemented to compute the sound transmission provided by single and double walls.

Steel and Craik [7] used both the SEA and the FEM to calculate the sound transmission between solid panels. The comparison of the results with measured data revealed that the FEM could be used for determining the coupling between subsystems, which are required when 
implementing the SEA model. Hynnä et al. [8] also used the SEA model to compute the structureborne sound transmission in large ship's structures. Pre-processing programs, frequently applied in the context of the FEM, were used to reduce the modelling work. Craik et al. [9] applied the SEA model to predict the sound transmission across double-leaf partitions, accounting for the vibration transmission across structural connections. Recently, Craik and Smith [10] revisited the problem of sound transmission through double-leaf lightweight partitions using a SEA model. In this work, the wall is modelled as a single subsystem at low frequencies, while at higher frequencies the SEA model utilizes a number of interconnected subsystems.

The FEM has been applied to analyze the effect of room dimension on the sound insulation of a separating panel at low frequencies [11,12]. The comparison of the results with experimental data [12] showed that the sound insulation is strongly dependent on the modal characteristics of the sound field within the adjacent rooms, at low frequencies.

Sgard et al. [13] predicted the diffuse field transmission loss through double walls with elastic porous linings (built as a porous-elastic decoupling material sandwiched between an elastic skin and a septum), at low frequencies, using a finite element model coupled to a variational BEM to account for fluid loading.

Kropp and Rebillard [14] used two models to calculate the sound insulation provided by a double wall at low frequencies. The first is a matrix formulation, which avoids the restrictions in the thickness of the layers that the Kirchhoff or Mindlin theories involve, taking the vibration properties on one side of a layer to be described by those on the other side. The second model simulates the double wall as two bending plates coupled by an elastic interlayer characterized by uncoupled springs, which ignores the contribution of the shear stiffness.

Fringuellino and Guglielmone [15] computed the transmission loss of multi-layered walls using a simplified approach based on the prior knowledge of the characteristic impedance of each material layer. Bolton et al. [16] described a theory for multi-dimensional wave propagation in elastic porous materials, based on Biot's theory, and used it to predict sound transmission through foam-lined panel structures. The transmission loss predictions of aluminium double panel structures lined with polyurethane foam were found to be in good agreement with laboratory experiments.

As it is quite difficult to predict sound insulation through single and double walls numerically and analytically, laboratory tests have been also carried out. Work by different authors $[17,18]$ has shown that the sound reduction index at low frequencies depends on several parameters, e.g., room dimension, the reverberation time and the position of sound source. Uris et al. [19] measured the influence of the bulk density of rock wool on the sound reduction index of double walls made from gypsum boards. The results indicated that for frequencies below $1250.0 \mathrm{~Hz}$ the sound reduction index can be increased by reducing the rock wool density, while for higher frequencies this parameter does not greatly affect the acoustic insulation.

This article predicts the sound reduction provided by an infinite double panel wall in the presence of a spatially sinusoidal harmonic line load or a plane pressure load. The work extends recent work by the authors [20], where analytical solutions for predicting the acoustic insulation provided by an infinite single homogeneous wall in the presence of spatially harmonic line loads were presented. This method does not impose limits on the thickness of the layer, as the Kirchhoff or Mindlin theories require, and it does consider the coupling between the fluid (air) and the solid panel. It follows the technique described by the authors in the calculation of the steady state 
response of a homogeneous three-dimensional slab and a solid elastic layer bounded by two fluid media subjected to spatially sinusoidal harmonic line loads [21,22].

This paper first briefly describes the analytical method used to compute the sound reduction of a double wall, subjected to a sinusoidal line pressure load. The analytical model is then used to compute the sound reduction provided by a double homogeneous brick wall, with identical panels, when struck by plane sound waves. These results are compared with those provided by the simplified method proposed by London, (later extended by Beranek). The different sound transmission mechanisms are illustrated by analyzing synthetic time signatures. Next, the effect of the cavity absorption on the acoustic insulation is defined by ascribing complex densities to the air filling the space between the two wall panels. Finally, a number of applications for different combinations of wall and air layer thickness are presented to assess the influence of this variable on the final acoustic insulation.

\section{Formulation of the method}

The model assumes the existence of two solid horizontal layer formations, with thickness $h_{1}$ and $h_{2}$, separated by a fluid layer with thickness $h_{3}$, and bounded by two external fluid media, excited by a spatially sinusoidal harmonic pressure load acting in the top fluid medium. The material properties of the two walls may differ, as may the properties of the three fluid media (see Fig. 1). This system is assumed to be excited by a spatially sinusoidal harmonic pressure load placed in the top fluid layer. The technique requires both the solid displacement potentials and fluid pressure potentials to be known. The solid displacement potentials were defined by the authors [23] when evaluating the Green functions for a harmonic (steady state) line load with a sinusoidally varying amplitude in the third dimension in an infinite medium, with $k_{z}$ being the wave number along this direction. A similar procedure is followed to obtain the fluid pressure potential. These potentials are then expressed as a superposition of plane waves, with different wave numbers, $k_{n}$, along the $x$ direction, following the technique used first by Lamb [24] for the two-dimensional case, and then by Bouchon [25] and Kim and Papageorgiou [26] to calculate the three-dimensional field by means of a discrete wave number representation. This formulation assumes the existence of an infinite

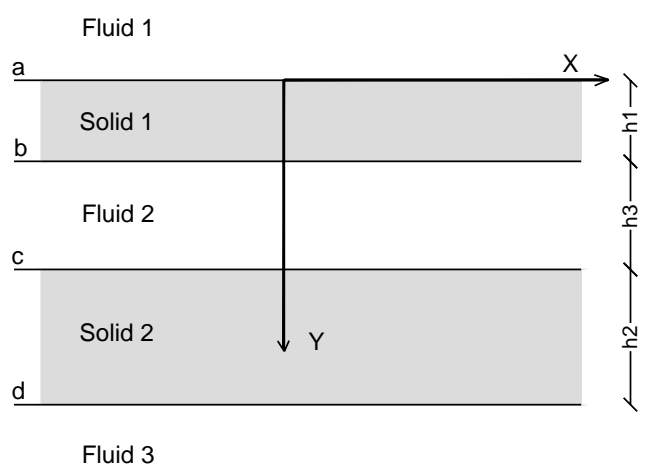

Fig. 1. Geometry of the model. 
number of virtual loads distributed along the $x$ direction, at equal intervals $L_{x}$, permitting the definition of $k_{n}=\left(2 \pi / L_{x}\right) n$.

The final solution is expressed as the sum of the source terms equal to those in the full-space and the surface terms needed to satisfy the boundary conditions, namely, continuity of normal displacements and stresses, and null tangential stresses conditions at the four fluid-solid interfaces ( $a, b, c$ and $d$, see Fig. 1). For this specific problem, all solid-fluid interfaces generate surface terms, which can be expressed in a form similar to that of the source term. The technique is similar to the one proposed by the authors for defining the acoustic insulation provided by a single wall [20]. The final system of equations is built using a scheme similar to that used in the Global Matrix formulation [27].

\section{Numerical applications}

The sound reduction provided by a double infinite extent wall subjected to plane pressure loads or harmonic line pressure loads has been computed using analytical expressions. The plane load is simulated, ascribing single values of $n$ and $k_{z}$, while the harmonic line load is calculated performing the full summation in $k_{n}$, following the technique defined by the authors [20]. The computations are first performed in the frequency domain, which allows the sound reduction to be obtained. Then, synthetic time signatures are determined by applying an inverse Fourier transform. The time variation of the load follows a Ricker wavelet pulse, defined in the frequency domain by

$$
U(\omega)=A\left[2 \sqrt{\pi} t_{0} \mathrm{e}^{-\mathrm{i} \omega t_{s}}\right] \Omega^{2} \mathrm{e}^{-\Omega^{2}},
$$

where $A$ is the amplitude, $\Omega=\omega t_{0} / 2, t_{s}$ is the time when the maximum occurs, while $\pi t_{0}$ is the characteristic (dominant) period of the pulse. The Fourier transformations are obtained by computing discrete summations over wave numbers and frequencies. Complex frequencies, with a small imaginary part of the form $\omega_{c}=\omega-$ i $\eta$ (with $\eta=0.7 \Delta \omega$ ), are used to avoid the aliasing phenomena [20].

In the examples, a ceramic double wall is used to illustrate the results. Different combinations of panel thickness and air cavity dimension were ascribed to the wall. The ceramic material has a shear modulus of $2.5 \mathrm{GPa}$, an elasticity modulus of $6.0 \mathrm{GPa}$, a Poisson coefficient of 0.20 and a density $\left(\rho^{s}\right)$ of $1400.0 \mathrm{~kg} / \mathrm{m}^{3}$, allowing a dilatational wave velocity $\left(\alpha^{s}\right)$ of $2182.2 \mathrm{~m} / \mathrm{s}$ and a shear wave velocity $\left(\beta^{s}\right)$ of $1336.3 \mathrm{~m} / \mathrm{s}$. The fluid media (air) allow a pressure wave velocity $\left(\alpha^{f}\right)$ of $340 \mathrm{~m} / \mathrm{s}$ and a density $\left(\rho^{f}\right)$ of $1.22 \mathrm{~kg} / \mathrm{m}^{3}$. The pressure response is computed over a grid of receivers placed on either side of the double wall, when the source is positioned $2.0 \mathrm{~m}$ away from the wall, as shown in Fig. 2.

The computations are performed with a frequency increment of $2 \mathrm{~Hz}$, from 2 to $8192 \mathrm{~Hz}$. The frequency increment used defines a total time window $T=0.5 \mathrm{~s}$ for the analysis. The spatial distance between the virtual point sources has been set at $4 \alpha^{s} T$ ( $\alpha^{s}$ is the dilatational wave velocity in the solid panels). Selected results are given to illustrate the main findings of this work. The first simulations use a double wall where the thickness of the first panel is $h_{1}=0.10 \mathrm{~m}$ and that of the second is $h_{2}=0.10 \mathrm{~m}$, separated by an air gap of $h_{3}=0.10 \mathrm{~m}$. The influence of the incidence angle of plane waves on the sound reduction is assessed by subjecting this wall to the incidence of plane 

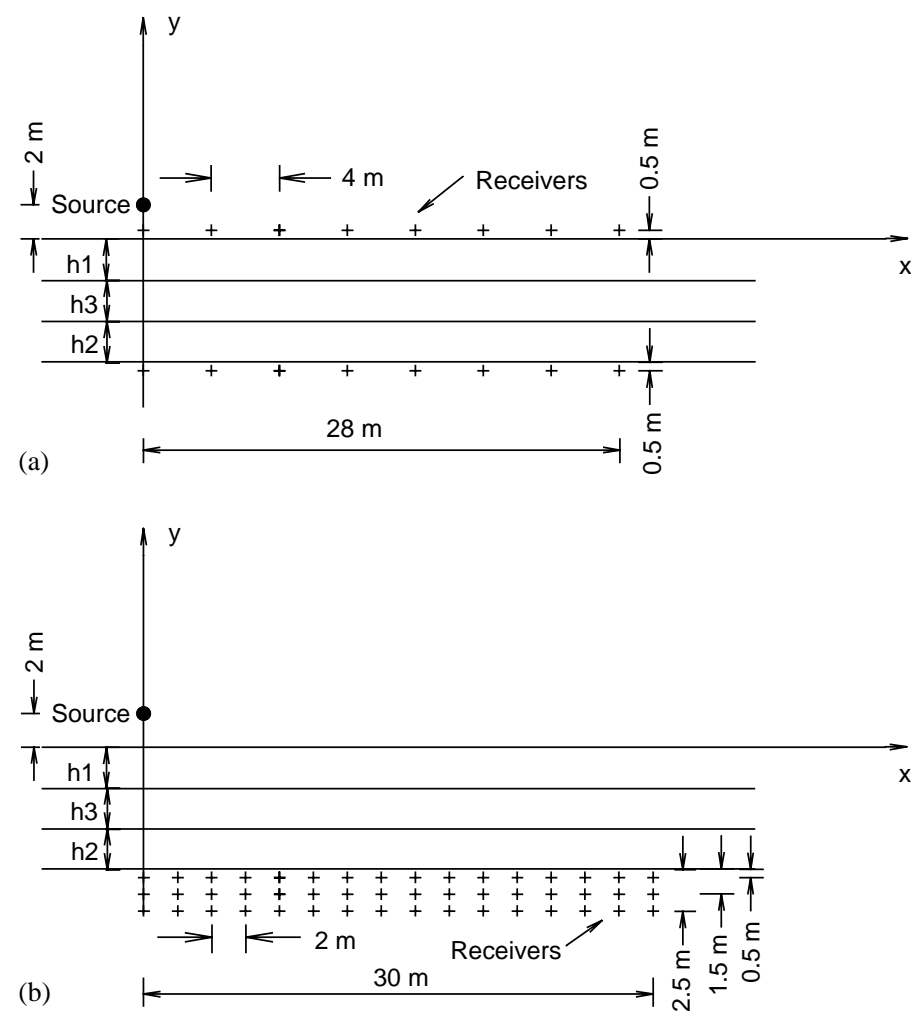

Fig. 2. Position of the receivers and sources: (a) time domain computations and (b) frequency domain computations.

waves with different apparent velocities. The results are compared with those provided by the simplified model proposed by the London-Beranek method. The effect of cavity absorption is also considered by using complex densities for the air layer separating the two solid panels. Different absorbing materials are modelled with different flow resistivity values. This wall is then subjected to the incidence of two-dimensional cylindrical waves $\left(k_{z}=0\right)$ to obtain responses in the time domain for different excitation frequency pulses. The different transmission mechanisms through the double wall are analyzed and identified in the time signatures. Additional simulations are performed, varying the thickness of the panels and the air cavity, to assess the influence of these parameters on the sound reduction when the walls are struck by cylindrical waves. Finally, the sound reduction curves obtained for double walls are compared with those provided by a single wall with identical mass.

\subsection{Incidence of plane waves}

A ceramic brick double wall, composed of two identical panels $0.10 \mathrm{~m}$ thick, with an air cavity of $0.10 \mathrm{~m}$, is subjected to a plane wave propagating perpendicularly to the direction of the wall panel at an apparent velocity of $v=\infty \mathrm{m} / \mathrm{s}$. The sound pressure is computed on the other exterior side of the double wall, in the medium not containing the source, along the grid of receivers 


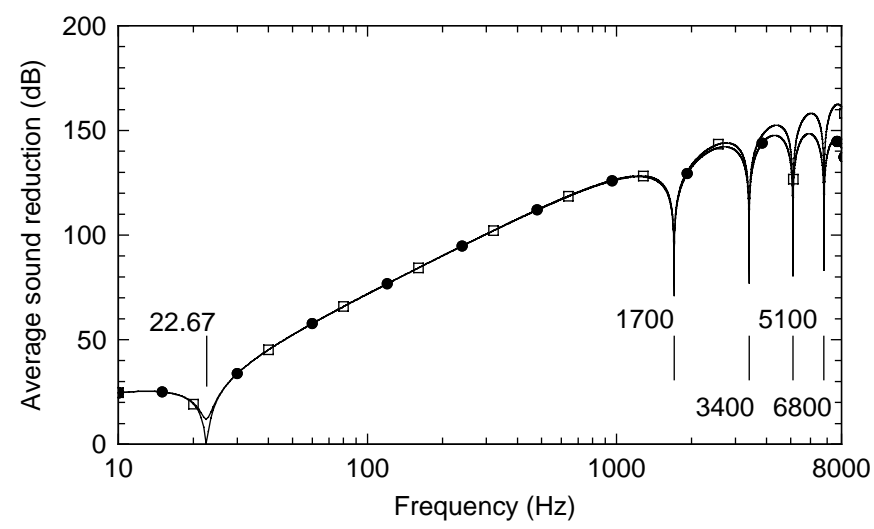

Fig. 3. Average sound reduction provided by a double ceramic brick wall composed of panels $10.0 \mathrm{~cm}$ thick and an air layer $10.0 \mathrm{~cm}$ thick, when subjected to normally incident plane waves: analytical model versus a simplified model $(\bullet-v=\infty \mathrm{m} / \mathrm{s}$; —-Eq. (2) $v=\infty \mathrm{m} / \mathrm{s})$.

defined in Fig. 2b. The computations are performed both with and without the presence of the double wall to assess the level of sound pressure reduction provided by the wall. The average sound reduction over the grid of receivers is displayed in Fig. 3, on a dB scale, for the conditions described above. The results provided by the London-Beranek method, are also plotted in this figure. This model defines sound reduction as

$$
R_{\phi}=10 \log \left[1+\left(\frac{\omega}{\omega_{0}}\right)^{2}\left(\frac{\omega_{0} M_{s}}{\alpha^{f} \rho^{f}}\right)^{2} \cos ^{2} \phi\left(\cos \zeta-\frac{1}{2} \frac{\omega}{\omega_{0}} \frac{\omega_{0} M_{s}}{\alpha^{f} \rho^{f}} \cos \phi \sin \zeta\right)^{2}\right],
$$

where

$$
\zeta=2 \frac{\omega}{\omega_{0}} \frac{\alpha^{f} \rho^{f}}{\omega_{0} M_{s}} \cos \phi, \quad \omega_{0}=\sqrt{\frac{2\left(\alpha^{f}\right)^{2} \rho^{f}}{h_{3} M_{s}}},
$$

$\phi$ is the angle of incidence of sound waves, $M_{s}$ is the mass of each panel $\left(\mathrm{kg} / \mathrm{m}^{2}\right)$ for equal panels and $h_{3}$ is the thickness of the air cavity (m).

The two curves exhibit similar behaviour. However, at higher frequencies the sound reduction obtained with Eq. (2) is larger than that given by the analytical model.

The mass-air-mass resonance frequency, calculated using the simplified expression

$$
f_{\text {res }}=\frac{\alpha^{f}}{2 \pi \cos \phi} \sqrt{\frac{\rho_{f}}{h_{3}}\left(\frac{1}{m_{1}}+\frac{1}{m_{2}}\right)},
$$

where $\phi$ is the angle of incidence of sound waves, and $m_{1}$ and $m_{2}$ are the mass of each panel $\left(\mathrm{kg} / \mathrm{m}^{2}\right)$, agrees with the position of the sound reduction dips defined by the two models. Similarly, the positions of sound reduction dips related to the resonance frequencies within the air layer $\left(f_{k}(\mathrm{~Hz})=\alpha^{f} k /\left(2 h_{3}\right), k=1,2, \ldots\right)$ are predicted well by both models. Notice that the coincidence phenomenon is not present in this particular case, since the incident field strikes the wall along the direction perpendicular to the wall plane, and does not generate waves travelling along the wall panels. 


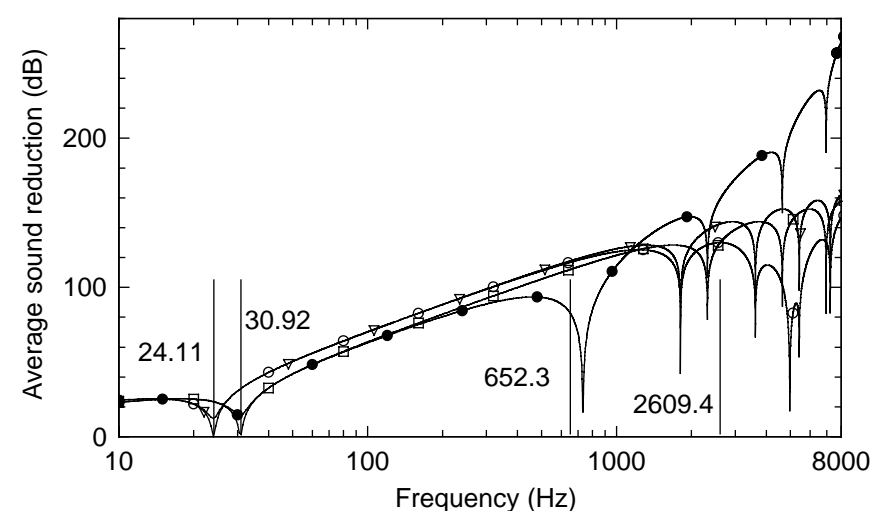

Fig. 4. Average sound reduction provided by a double ceramic brick wall composed of panels $10.0 \mathrm{~cm}$ thick and an air layer $10.0 \mathrm{~cm}$ thick, when subjected to obliquely incident plane waves: analytical model versus a simplified model ( $\mathrm{O}$ $v=1000 \mathrm{~m} / \mathrm{s} ; \bullet-v=500 \mathrm{~m} / \mathrm{s} ; \nabla \longrightarrow$ Eq. (2) $v=1000 \mathrm{~m} / \mathrm{s} ; \square-$ Eq. (2) $v=500 \mathrm{~m} / \mathrm{s}$ ).

Fig. 4 shows the sound reduction provided by the same wall, on a dB scale, when subjected to plane waves with differing incidence angles defining different apparent wave velocities, namely $v=1000.0$ and $500.0 \mathrm{~m} / \mathrm{s}$. These calculations were performed ascribing $k_{z}=0$ and defining $n$ in such a way as to model the different plane waves. Thus, $v=1000.0 \mathrm{~m} / \mathrm{s}$ correspond to plane waves reaching the surface with inclination of $19.9^{\circ}$, while $v=500.0 \mathrm{~m} / \mathrm{s}$ correspond to plane waves reaching the surface with inclination of $42.8^{\circ}$ in relation to the normal direction of the wall.

Additional curves, representing the results obtained by Eq. (2), are also plotted in Fig. 4. Comparing the analytical curves with the simplified model curves some important differences emerge. The analytical model predicts dips of sound reduction due to the coincidence effect, which are not taken into account by the simplified model. The coincidence effect occurs when the wavelength of the sound waves projected on a panel equals the wavelength of guided waves travelling along the wall panels, leading to increased movement of the panel, which causes low sound insulation.

The propagation of these guided waves is frequently analyzed without taking the presence of fluid on the two faces of each panel into account. The mathematical development of these assumptions leads to the following dispersion relation [28]:

$$
c_{L}=\left(\frac{D \omega^{2}}{\rho h}\right)^{0.25},
$$

where $c_{L}$ is the phase velocity of the propagating plane waves along the plate, $\rho$ is the density of the material $\left(\mathrm{kg} / \mathrm{m}^{3}\right), h$ is the thickness of the panel (m), $\omega=2 \pi f, D=h^{3} E /\left[12\left(1-v^{2}\right)\right]$ with $E$ and $v$, being the Young's modulus and the Poisson ratio, respectively. The mathematical manipulation of this equation leads to the expression

$$
\omega=\left(\frac{\alpha^{f}}{\sin \phi}\right)^{2} \sqrt{\frac{\rho h}{D}},
$$

where $\phi$ is the incidence angle of the sound relative to a direction perpendicular to the element. This assumption does not introduce significant misinterpretations in the insulation predictions 
because the sound waves travel at a much slower velocity than the body wave velocities in the plate. The critical frequency $\left(f_{c}\right)$ is taken to correspond to $\phi=90^{\circ}$,

$$
f_{c}=\frac{\left(\alpha^{f}\right)^{2}}{1.8138 h} \sqrt{\frac{\rho\left(1-v^{2}\right)}{E}} .
$$

The differences between the results computed by Eq. (5), for the frequencies associated with the plane waves travelling along the plate at velocities of $v=500.0$ and $1000.0 \mathrm{~m} / \mathrm{s}(f=652.3$ and $2609.4 \mathrm{~Hz}$ ), and those of the insulation dips provided by our model grow as the inclination of the waves decreases.

Comparing the results of the two models again, one finds they agree when the frequency is very low, far below the frequency associated with the coincidence effect. As the frequency increases the responses become very different, particularly when the apparent wave velocity decreases. The two models predict identical frequency positions for the dips associated with the air layer resonance frequencies, which are now dependent on the angle of incidence of the plane waves $\left(f_{k}(\mathrm{~Hz})=\alpha^{f} k /\left(2 h_{3} \cos \phi\right), k=1,2, \ldots\right)$.

The effect occurring when absorbing material fills the air layer between the two solid panels is addressed using the approach proposed by Fahy [6]. This effect is thus taken into account by using a complex air density, which depends on the flow resistivity and porosity of the material and on a structure factor. The complex density is computed as

$$
\rho_{f}^{\prime}=s \rho_{f} \vartheta-\mathrm{i} \sigma / \omega,
$$

where $s$ is a structure factor, $\vartheta$ is the porosity and $\sigma$ is the flow resistivity. In our model, $s$ and $\vartheta$ are assumed to be one. The complex fluid wave number is computed accordingly.

In the analytical results presented, the air cavity is assumed to be filled with mineral wool with densities of $50.0,70.0,100.0$ and $200.0 \mathrm{~kg} / \mathrm{m}^{3}$, which correspond to flow resistivities of 1000.0 , $2000.0,3144.0$ and $10000.0 \mathrm{~kg} / \mathrm{m}^{3} / \mathrm{s}$, respectively [29]. A plane wave strikes the double wall along the direction perpendicular to its plane. The results are displayed in Fig. 5. The absorption has a

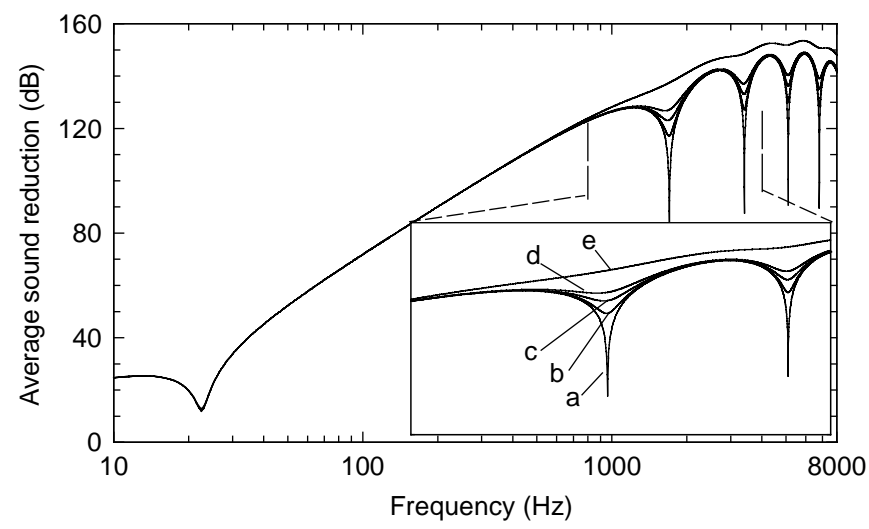

Fig. 5. Average sound reduction provided by a double ceramic brick wall composed of panels $10.0 \mathrm{~cm}$ thick and an air layer $10.0 \mathrm{~cm}$ thick, filled with different absorbing materials, when subjected to normally incident plane waves: (a) $\sigma=0 \mathrm{~kg} / \mathrm{m}^{3} / \mathrm{s}^{1}$; (b) $\sigma=1000 \mathrm{~kg} / \mathrm{m}^{3} / \mathrm{s}$; (c) $\sigma=2000 \mathrm{~kg} / \mathrm{m}^{3} / \mathrm{s}$; (d) $\sigma=3140 \mathrm{~kg} / \mathrm{m}^{3} / \mathrm{s}$; and (e) $\sigma=10000 \mathrm{~kg} / \mathrm{m}^{3} / \mathrm{s}$. 
weak effect on the mass-air-mass resonance dip, but attenuates the dips of insulation controlled by the cavity resonances.

\subsection{Incidence of cylindrical waves}

Next, the double wall is subjected to the incidence of cylindrical waves of $k_{z}=0$, which can be viewed as a pure two-dimensional linear source.

\subsubsection{Time responses}

Time signatures have been calculated simulating the wave propagation in the vicinity of the ceramic brick double wall used above, that is, with panels $10.0 \mathrm{~cm}$ thick and a $10.0 \mathrm{~cm}$ air layer. The results are calculated at receivers placed $0.5 \mathrm{~m}$ from the wall surface, on both sides of the wall (see Fig. 2a).

The wall is excited by waves generated by a source placed $2.0 \mathrm{~m}$ away from the wall surface, emitting a Ricker pulse with a characteristic frequency of $3000 \mathrm{~Hz}$. Fig. 6a displays the response in the fluid medium containing the source. Each time signature clearly shows two pulses that correspond to the direct incident pulse, and a pulse arriving later at the receivers, after being reflected on the surface wall. Notice that the responses contain additional pulses, produced by refractions and mode conversions at the wall interface and guided waves that travel along the wall. The scaling of Fig. 6a makes it difficult to distinguish these waves.

Fig. $6 \mathrm{~b}$ shows the time responses obtained in the fluid medium on the other side of the wall. The response amplitudes suffer a pronounced drop compared with those computed in the medium containing the source. This behaviour was expected, given the insulation provided by the wall. The response appears more complex. However, the features of this plot are similar to the ones that are not easily visible in Fig. 6a, due to the scale of the plot. A pack of high-frequency pulses is clearly visible after the arrival of the first dilatational waves, followed by a ring of low-frequency waves, up to the arrival of the slower $P$ body pulses. Analysis of the responses shows that the receiver, placed at the same abscissa as the source $(x=0.0 \mathrm{~m})$, does not record the existence of guided waves. Indeed, these waves are dispersive and appear some way from the source. Furthermore, the results show the contribution of pulses that originate in the mass-air-mass vibration and in the resonance frequencies within the air layer. This wave field is recorded along the whole set of receivers. It is not clear at the first receiver, placed at $x=0.0 \mathrm{~m}$, because of the scale of the Fig. $6 \mathrm{~b}$ plot.

To illustrate the sound transmission mechanisms that occur at $x=0.0 \mathrm{~m}$ better, Fig. $6 \mathrm{c}$ gives the computed response using an appropriate scale. The response has a periodic behaviour, exhibiting a regular time period between successive peaks and valleys, approaching the period associated with the mass-air-mass resonance frequency

$$
\left(1 / f_{\text {res }}=1 / \frac{\alpha^{f}}{2 \pi} \sqrt{\frac{\rho^{f}}{h_{3}}\left(\frac{1}{m_{1}}+\frac{1}{m_{2}}\right)}=44.1 . \mathrm{ms}\right) .
$$

However, this low-frequency harmonic behaviour of the response is built up by the superposition of higher frequency periodic responses related to the resonance frequencies within the air layer $\left(T=2 h_{3} / \alpha^{f}=0.59 \mathrm{~ms}\right)$. 



Fig. 6. Synthetic wave forms at receivers placed $0.5 \mathrm{~m}$ away from a double ceramic brick wall, composed of panels $10.0 \mathrm{~cm}$ thick and an air layer $10.0 \mathrm{~cm}$ thick subjected to a cylindrical dilatational Ricker pulse source with a characteristic frequency $3000 \mathrm{~Hz}\left(k_{z}=0\right)$ : (a) in the fluid medium containing the source; (b) in the exterior fluid medium not containing the source; and (c) in the exterior fluid medium not containing the source at $x=0.0 \mathrm{~m}$. 
At excitation frequencies below the first resonance frequency of the air cavity $(1700 \mathrm{~Hz})$, this signal would not exhibit the periodic behaviour related to the resonance frequencies of the air layer (not illustrated).

\subsubsection{Frequency responses}

Fig. 7 plots the average sound reduction, on a $\mathrm{dB}$ scale. The results clearly show the dips related to the coincidence effect, the mass-air-mass resonance and resonance frequencies of the air layer. The calculated average sound reduction, when the air cavity is filled with mineral wool with a density of $50.0 \mathrm{~kg} / \mathrm{m}^{3}$, which corresponds to a flow resistivity of $1000.0 \mathrm{~kg} / \mathrm{m}^{3} / \mathrm{s}^{1}$, is also displayed. The results reveal that the sound insulation provided by the wall, when there is mineral wool in the air layer, increases, particularly at frequencies below the coincidence effect and above the mass-air-mass resonance frequency. As with the plane waves, sound insulation is also greater in the vicinity of the insulation dips related to the cavity resonances. However, the position of these resonance frequencies is affected by pressure waves that travel within the air layer with different path inclinations. So, these dips of insulation appear later in frequency than those found when the source emits a plane wave striking the wall perpendicularly.

\subsection{Importance of panel mass and air gap thickness}

A set of simulations is presented with varying panel and air cavity thickness to assess the influence of these parameters on the sound reduction. The different plots displayed below are labelled using indexes $a-b-c$ (in $\mathrm{cm}$ ): the indexes $a$ and $c$ indicate the thickness of the first (on the source side) and second panel, respectively, while the index $b$ relates to the thickness of the air layer.

The effect of the air layer thickness on the sound reduction is addressed first. The thickness of the ceramic panels remains $10.0 \mathrm{~cm}$, while the air layer assumes a thickness of 5.0, 10.0 and $20.0 \mathrm{~cm}$. Fig. 8 illustrates the results obtained when the double wall is illuminated by a cylindrical

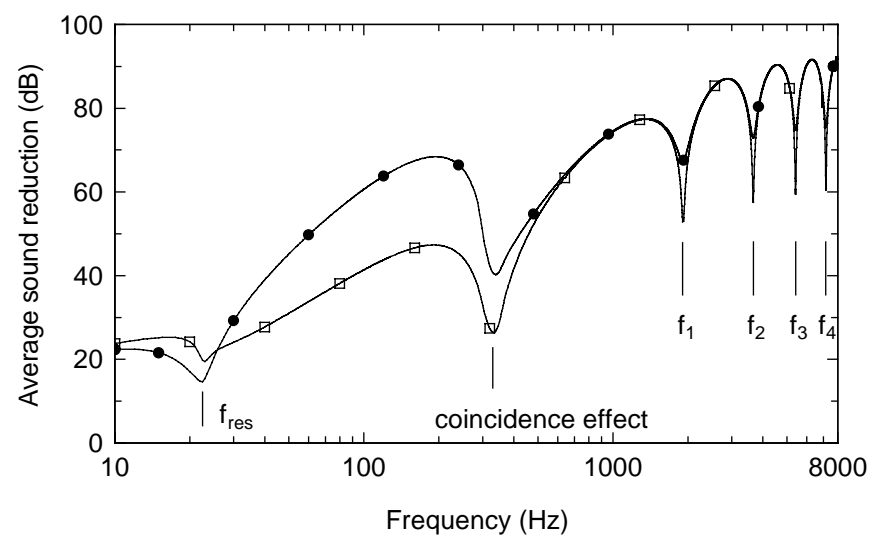

Fig. 7. Sound transmission loss provided by a double ceramic brick wall composed of panels $10.0 \mathrm{~cm}$ thick and an air layer $10.0 \mathrm{~cm}$ thick, filled with an absorbing material, when subjected to a cylindrical linear source $\left(k_{z}=0\right)$ $\left(\square-\sigma=0 \mathrm{~kg} / \mathrm{m}^{3} / \mathrm{s} ; \bullet-\sigma=1000 \mathrm{~kg} / \mathrm{m}^{3} / \mathrm{s}\right)$. 


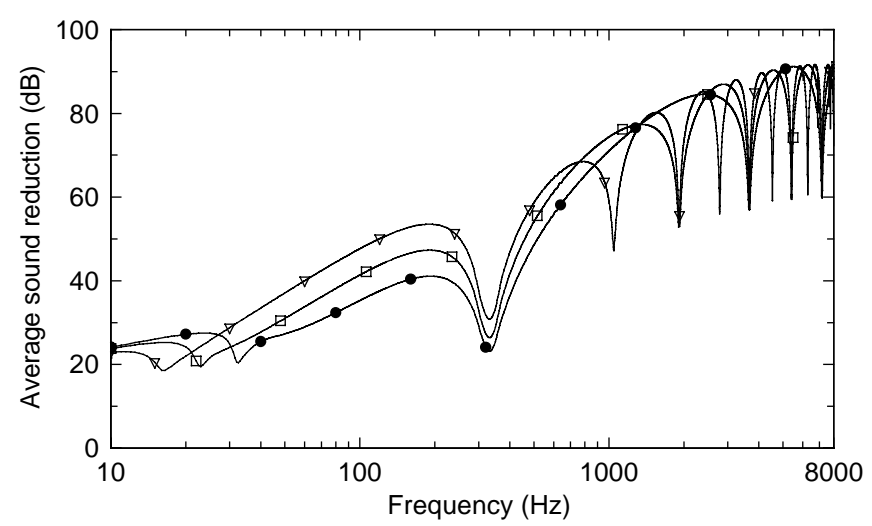

Fig. 8. The effect of the air layer thickness on the sound reduction when a double ceramic wall, with panels $10 \mathrm{~cm}$ thick, is subjected to the incidence of cylindrical waves with wave velocity along the $z$ direction. $v_{z}=\infty \mathrm{m} / \mathrm{s}\left(k_{z}=0\right)(\bullet-10$ 5-10; 口-10-10-10; $\nabla-10-20-10)$.

linear source, $k_{z}=0$. At frequencies above the mass-air-mass resonance frequency, and below the effect of the air layers resonance frequencies, the sound reduction increases as the air layer thickness grows. For high frequencies the sound reduction is dominated by the influence of the air cavity resonances. As the air cavity thickness decreases, the gap between dips associated with the air cavity resonances increases, and so does the frequency for which the dip occurs.

Fig. 9 illustrates the sound reduction, on a $\mathrm{dB}$ scale, computed when the air gap between the two panels is kept at a constant thickness of $5.0 \mathrm{~cm}$, while the two panels change their individual thickness but maintain the same global mass (19-5-1, 16-5-4, 15-5-5, 14-5-6, 12-5-8, 10-5-10 and 5-5-15). The results are given in three different plots. The results for the double wall 15-5-5 are shown in all plots to allow a straight comparison of the different results.

Fig. 9a displays a first set of results corresponding to three different configurations of the double wall: $15-5-5,10-5-10$ and 5-5-15. The results suggest a poorer performance when the wall is built with identical mass panels, particularly at high frequencies. When the mass of the two panels is different, the results are not influenced by the order of the panels. In this case, two dips related to the coincidence effect are clearly visible, and are associated with each wall panel.

Fig. $9 \mathrm{~b}$ presents the sound reduction provided by the walls defined as 15-5-5, 14-5-6 and 12-5-8. As expected, the position of the sound reduction dips associated with the coincidence effect and the mass-air-mass resonance frequencies changes according to the thickness of the two panels, as suggested by Eqs. (3) and (6). The sound reduction dips related to the resonance frequencies within the air layer occur at the same frequencies for all cases, given the presence of constant air layer thickness. Fig. 9c gives the results for simulations for walls 19-5-1, 16-5-4 and 15-5-5. As before, dips of insulation associated with the coincidence effect and the resonance within the air layer occur. However, as the thickness of one panel increases, the responses exhibit additional dips related to the resonance effects within the solid layer. This phenomenon can be identified at high frequencies when the wall is defined by 19-5-1, markedly affecting the response plotted by the sound reduction curve. Notice that the same behaviour occurs for the thinner walls, but at higher frequencies. When one of the panels is 0.14 or $0.16 \mathrm{~m}$, this effect occurs in the vicinity of the frequencies associated with the resonance frequencies within the air layer, and thus cannot be 



Fig. 9. Influence of the mass of each panel on the sound reduction provided by a double wall, when the air gap and the global mass are kept constant, when the wall is subjected to a cylindrical linear source: (a) $\bullet-5-5-15$; $\square-10-5-10$; $\nabla-$ 15-5-5 and (b) ○-12-5-8; 口-14-5-6; $\nabla-15-5-5$; (c) $\nabla-15-5-5$; $\bullet-16-5-4$; $\square-19-5-1$. 


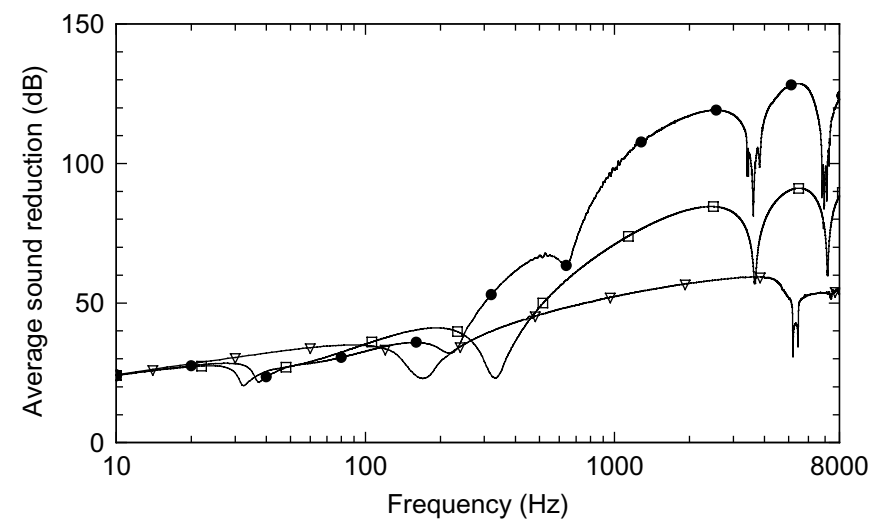

Fig. 10. Comparison of the sound reduction performance provided by double walls with that guaranteed by single walls with same global mass $(\bullet-15-5-5$; $\square-10-5-10 ; \nabla-$ single wall -20$)$.

easily identified. For thinner walls, this phenomenon is observed at higher frequencies outside the domain being analyzed.

Fig. 10 compares the performance of double walls with that of a single panel wall with the same mass. The sound reduction curve provided by the single panel wall exhibits the dip of insulation caused by the coincidence effect. At very high frequencies, a dip related to the interaction of waves within the solid panel can be seen, as described above. Comparing this curve with those obtained for double walls indicates that higher insulation is achieved for double walls, particularly when the two panels have different masses. This behaviour is more pronounced for higher frequencies.

\section{Conclusions}

The steady state pressure response generated by a spatially sinusoidal harmonic line load, illuminating a double panel wall, was obtained analytically. The analytical solutions enabled the sound pressure reduction provided by a double wall to be computed when it is subjected to plane and cylindrical pressure waves. The presence of an absorbing material within the air cavity may be taken into account by ascribing a complex density to the air filling the cavity. Time signatures were computed using inverse Fourier transformations, to better understand the sound transmission mechanisms through a double wall.

The sound reduction computed using the proposed model was compared with that calculated using the simplified model referred to as the London-Beranek model.

The synthetic time signals computed in the exterior fluid media reveal the presence of an intricate wave field. This wave field is caused by body and guided surface waves, multiple reflections within the solid and fluid layers and the global resonance of the system. The guided waves associated with the coincidence effects were found to be very dispersive, with faster phase velocities related to higher excitation frequencies. Analysis of the arrival times of the different pulses and their periodicity revealed the influence of all sound transmission mechanisms. The use of sources excited at low characteristic frequencies does not allow the excitation of some of these mechanisms, such as the ones associated with air cavity resonances. 
Different simulations were performed to obtain the sound reduction for different thicknesses of wall panels and air cavities, while keeping the total mass of the wall constant. The influence of the air cavity on the sound reduction was found to be dependent on the frequency. At low frequencies a better performance was achieved for thicker air layers, while at higher frequencies a thinner air layer is preferable. The use of wall panels with different mass resulted in the wall performing better, particularly for high frequencies, even when there was an additional dip related to the coincidence effect. The order of the panels was not found to be an important factor influencing the sound reduction, when the double wall is built with panels of different thicknesses. At high frequencies, however, the results seem to be affected by resonance phenomena within the solid panels. This is particularly important for thicker panels since this phenomenon is observed at lower frequencies.

Additional simulations confirmed that the sound reduction provided by a double wall is markedly superior when compared with that provided by a single wall with the same global mass. This tendency was more evident at high frequencies.

\section{References}

[1] A.C.C. Warnock, W. Fasold, Sound Insulation: Airborne and Impact, Encyclopedia of Acoustics, Vol. 3, WileyInterscience, New York, 1997, pp. 1129-1161.

[2] I.I. Novikov, Low-frequency sound insulation of thin plates, Applied Acoustics 54 (1998) 83-90.

[3] A. London, Transmission of reverberant sound through double walls, Journal of the Acoustical Society of America 22 (1950) 270-279.

[4] L.L. Beranek (Ed.), Noise Reduction, McGraw-Hill, New York, 1960.

[5] K. Gösele, Zur Berechnung der Luftschalldämmung von doppelschaligen Bauteilen (ohne Verbindung der Schalen), Acustica 45 (1980) 218-227.

[6] F. Fahy, Foundations of Engineering Acoustics, Academic Press, New York, 2001.

[7] J.A. Steel, R.J.M. Craik, Statistical energy analysis of structure-borne sound transmission by finite element methods, Journal of Sound and Vibration 178 (4) (1994) 553-561.

[8] P. Hynnä, P. Klinge, J. Vuoksinen, Prediction of structure-borne sound transmission in large welded ship structures using statistical energy analysis, Journal of Sound and Vibration 180 (4) (1995) 583-607.

[9] R.J.M. Craik, T.R.T. Nightingale, J.A. Steel, Sound transmission trough a double leaf partition with edge flanking, Journal of the Acoustical Society of America 101 (2) (1997) 964-969.

[10] R.J.M. Craik, R.S. Smith, Sound transmission through double leaf lightweight partitions Part I: airborne sound, Applied Acoustics 61 (2000) 223-245.

[11] A. Osipov, P. Mees, G. Vermeir, Numerical simulation of airborne sound transmission at low frequencies: the influence of the room and the partition parameters, Proceedings of Inter-Noise 2 (1997) 759-762.

[12] S.P.S. Maluski, B.M. Gibbs, Application of a finite-element to low-frequency sound insulation in dwellings, Journal of the Acoustical Society of America 108 (4) (2000) 1741-1751.

[13] F.C. Sgard, N. Atalla, J. Nicolas, A numerical model for the low frequency diffuse field sound transmission loss of double-wall sound barriers with elastic porous linings, Journal of the Acoustical Society of America 108 (6) (2000) 2865-2872.

[14] W. Kropp, E. Rebillard, On the air-borne sound insulation of double wall constructions, Acustica-Acta Acustica 85 (1999) 707-720.

[15] M. Fringuellino, C. Guglielmone, Progressive impedance method for the classical analysis of acoustic transmission loss in multilayered walls, Applied Acoustics 59 (2000) 275-285.

[16] J.S. Bolton, N.-M. Shiau, Y.J. Kang, Sound transmission through multi-panel structures lined with elastic porous materials, Journal of Sound and Vibration 191 (3) (1996) 317-347. 
[17] B. Berglund, P. Hassmen, R.F. Soames, Sources and effects of low-frequency noise, Journal of the Acoustical Society of America 99 (5) (1996) 2985-3002.

[18] W. Kropp, A. Pietrzyk, T. Kihlman, On the meaning of the sound reduction index at low frequencies, Acta Acustica 2 (1994) 379-392.

[19] A. Uris, A. Llopis, J. Llinares, Effect of rockwool bulk density on the airborne sound insulation of lightweight double walls, Applied Acoustics 58 (1999) 327-331.

[20] A. Tadeu, J. António, Acoustic insulation of single panel walls provided by analytical expressions versus the mass law, Journal of Sound and Vibration 257 (3) (2002) 457-475.

[21] A. Tadeu, J. António, 2.5D Green's functions for elastodynamic problems in layered acoustic and elastic formations, Journal of Computer Modeling in Engineering and Sciences 2(4) (2001) 477-495.

[22] A. Tadeu, J. António, Green's functions for 2.5D elastodynamic problems in a free solid layer formation, Journal of Engineering \& Structures 24(4) (2002) 491-499.

[23] A.J.B. Tadeu, E. Kausel, Green's functions for two-and-a-half dimensional elastodynamic problems, Journal of Engineering Mechanics, American Society of Civil Engineers 126 (10) (2000) 1093-1097.

[24] H. Lamb, On the propagation of tremors at the surface of an elastic solid, Philosophical Transactions of the Royal Society of London Series A 203 (1904) 1-42.

[25] M. Bouchon, Discrete wave number representation of elastic wave fields in three-space dimensions, Journal of Geophysical Research 84 (1979) 3609-3614.

[26] J. Kim, A.S. Papageorgiou, Discrete wavenumber boundary element method for 3-D scattering problems, Journal of Engineering Mechanics, American Society of Civil Engineers 119 (3) (1993) 603-624.

[27] F. Jensen, W. Kuperman, M. Porter, H. Schmidt, Computational Ocean Acoustics, Springer, Berlin, 2000.

[28] K.F. Graff, Wave Motion in Elastic Solids. Dover, New York, 1975.

[29] L.L. Beranek, I.L. Vér (Eds.), Noise and Vibration Control Engineering, Wiley, New York, 1992. 